\title{
Research article \\ Evaluation of changes seen in TMJ after mandibular advancement in treatment of Class II malocclusions, with functional appliances, a CBCT study
}

\author{
Parvathy Ram Mohan ${ }^{1}$, Sandeep Shetty ${ }^{2}$, Katheesa Parveen ${ }^{3}$ \\ ${ }^{1}$ Post Graduate Student, ${ }^{2}$ Professor, ${ }^{3}$ Senior Lecturer, Department of Orthodontics and Dentofacial Orthopedics, Yenepoya \\ Dental College, Yenepoya (Deemed to be) University, Mangalore Karnataka, India
}

(Received: March $2021 \quad$ Revised: April $2021 \quad$ Accepted: May 2021)

Corresponding author: Katheesa Parveen. Email: drkatheesaparveen@gmail.com

\begin{abstract}
Introduction and Aim: Various functional appliances are developed to remodel the glenoid fossa and, thereby change the position of the mandible and correct Class II malocclusion. Many diagnostic aids have already been used to evaluate the changes ,but Cone Beam computed tomography (CBCT), three -dimensional diagnostic tool is rarely used .So this study was conducted with an aim is to evaluate the changes seen in the temporomandibular joint (TMJ) after mandibular advancement in class II malocclusions with functional appliance therapy, using CBCT, and also to assess and compare the efficacy of Twin Block, Power Scope and Forsus Fatigue Device used for the correction of class II malocclusion.
\end{abstract}

Materials and Methods: The retrospective study was performed using the $52 \mathrm{CBCT}$ scans of 26 patients with class II malocclusion due to retrognathic mandible. The subjects were divided into three groups based on appliance (Twin Block, Power Scope and Forsus Fatigue Resistant Device) used for the treatment. The changes seen in TMJ after mandibular advancement and condylar response to functional therapy was evaluated using the six. Data obtained were subjected to one-way Anova analysis with Posthoc Tukey test. Software SPSS version 20 was used to analyse the data. The level of significance was set at $\mathrm{p} \leq 0.05$.

Results: In subjects treated with Twin Block, the condyle was displaced slightly downwards and anteriorly; and condylar height was reduced. The decrease in the anterior and superior joint spaces were also found. In subjects treated with PowerScope and Forsus Fatigue Resistant Device, there was an increase in condylar height and width with a decrease in anterior and superior joint space.

Conclusion: All three appliances were found to bring about changes in TMJ structures, but amongst them the Twin block has proved to be more efficient.

Keywords: TMJ; functional appliances; condylar changes; CBCT.

\section{INTRODUCTION}

$\mathrm{T}$ he term "functional appliance" refers to the various removable and fixed appliances that are designed to bring changes in the development, function and position of the mandible. This is obtained by changing the position of the mandible in anteroposterior and vertical direction, thus resulting in orthodontic and orthopaedic changes (1). Functional appliances are designed with an intent of increasing the length of mandible by positioning the mandible forward and holding it in a protruded position for several months. Consequently, condyle moves anteriorly. There is various research work done in humans reporting that the functional appliance brings the change in glenoid fossa by shifting it anteriorly and inferiorly; and also resulting in minor increase in mandibular length and posterior redirection of condylar growth (2). Increased cellular activity was also seen in TMJ with the use of fixed functional appliance on various animal experiments (3).

Cone beam computed tomography (CBCT) is a relatively latest growth in the ever-expanding horizon of clinical CT technologies. In this medical imaging technique, a cone-shaped X-ray beam is used instead of a collimated fan beam, with spiral CT centred on two-dimensional (2D) detectors producing a series of 2D images. The tube detector system uses constant beam and carries out a circuit around the patients' head. This rotation produces the initial data, which is used for primary reconstruction and these primary images are used for further secondary reconstructions in all planes i.e., three- dimensional (3D) reconstructions. The data obtained are rebuilt in 3D data set making use of the modified Feldkamp algorithm. Therefore, data are reconstructed in a volume than the slices, thus displaying 3D images. It also permits a multiplanar reformation i.e., 2D images in all the three planes (4).

In orthodontic literature, various imaging tools like cephalograms, panoramic radiographs, computed tomography and magnetic resonance imaging have been used to analyse condyle -fossa changes after mandibular advancement using various functional appliances. However, there are many limitations to these imaging techniques. The introduction of CBCT specifically in the oral and maxillofacial region brings a true paradigm shift from a $2 \mathrm{D}$ to a $3 \mathrm{D}$ approach in data acquisition and image reconstruction (3). Hence, 
CBCT is considered to be more adequate than conventional with many advantages (5-7). Several studies have analysed condyle-fossa modification after functional appliance therapy. Most of these studies have used lateral cephalograms, MRI or computed tomography to evaluate the changes following fixed functional treatment but these imaging techniques used have their own limitations. CBCT is the most accurate tool to reproduce the TMJ complex in three dimensions (3D) and overcomes the majority of the limitations of the above-mentioned tools. The data collected are reproducible and outstandingly more precise than those obtained with the conventional cephalograms.

\section{MATERIALS AND METHODS}

This study was conducted on the 52 CBCT scans of the patients that reported to the Department of Orthodontics for treatment and the ethical approval to analyse the retrospective data was obtained from the University with the protocol number YUEC003/03/01/2015 dated on 03/01/2015. The subjects were divided into 3 groups based on the appliance that was given to them. Group 1 was treated with Twin Block $(n=10)$, the second and third group of patients were treated with PowerScope (American Orthodontics, Washington $)(\mathrm{n}=10)$ and Forsus $^{\mathrm{TM}}$ Fatigue Resistant Device (3M Unitek Corp, Monrovia, Calif) $(n=6)$ respectively. The condylar response to functional therapy was evaluated on CBCT images that had been taken just before placement of the appliances and was compared to images taken after the appliance was removed.
The inclusion criteria were, that all the CBCT images of the patients were between 11 to 19 years and had a class II skeletal relationship due to a deficient mandible. CBCT images of the patients with class III skeletal malocclusion, age more than 20 years, syndromic and cleft lip and palate were excluded.

The Planmeca Promax 3D Mid was the equipment used for obtaining CBCT images pre and post treatment. The patient was made to sit in Natural Head Position during the exposure. Each exposure was done with a full FOV and the Dolphin 3D Imaging software was used to measure the condylar changes from three types of appliances used. Measurements were calculated keeping the external auditory meatus as a fixed landmark. Six parameters were chosen for the evaluation of the changes in TMJ structures that is condylar position, condylar height, condylar width, posterior articular space, superior articular space and anterior articular space. Measurements were made on both right and left condyles.

The CBCT images were saved in the DICOM format. The X-Ray images are later extracted, and the amount of hard tissue and soft tissue components were adjusted within the frame [Fig. 1]. In the Dolphin software, once the hard tissue component was finalized for the study, the TMJ region was isolated with the help of the horizontal reference plane. The slice thickness was maintained at $1 \mathrm{~mm}$. Isolating the TMJ width at $40 \mathrm{~mm}$ in the sagittal section with 6 cuts seen in the frame [Fig. 2]. As shown in fig 3, the yellow lines indicate the areas measured and the red lines denote the reference planes.

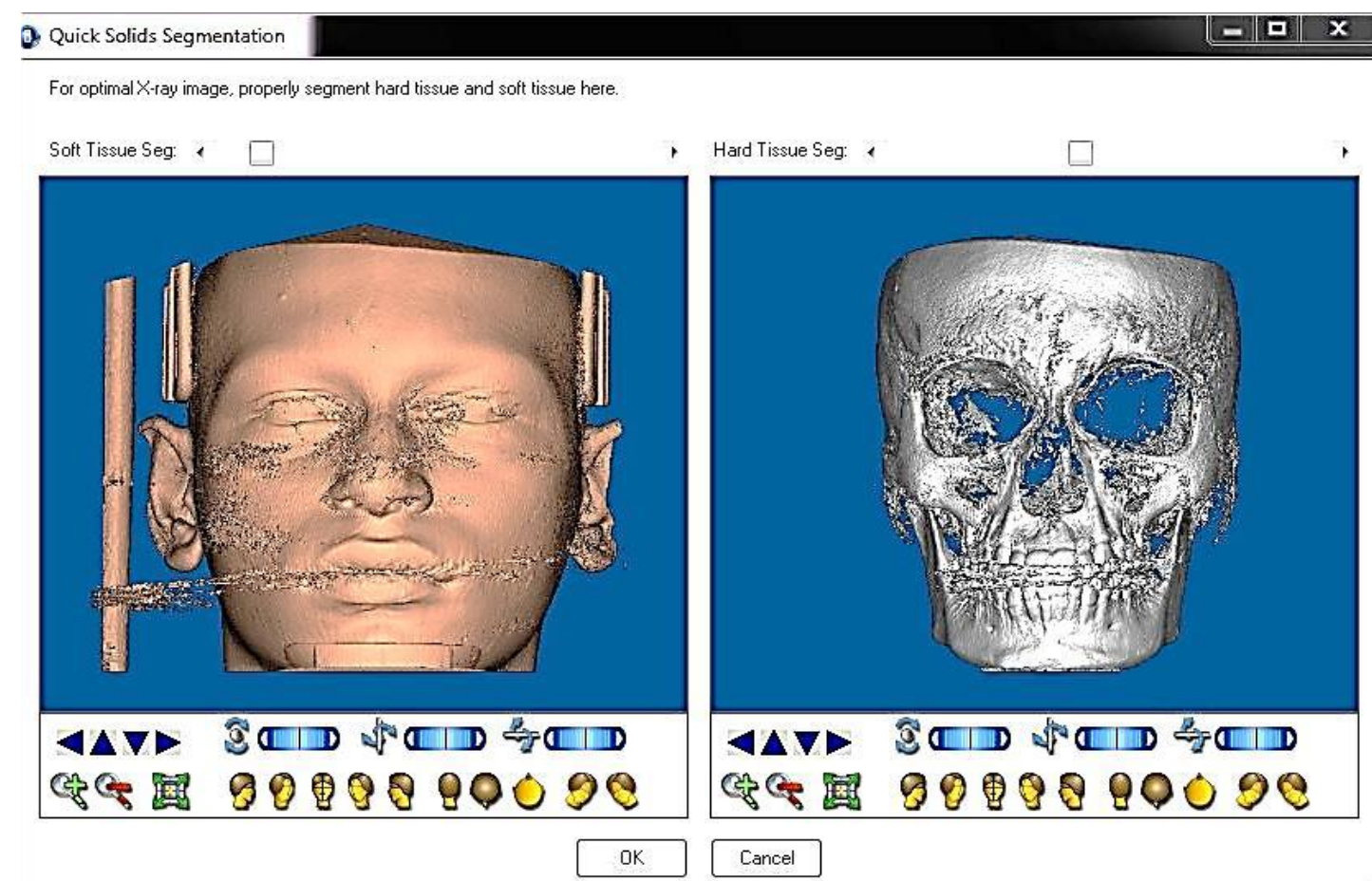

Fig. 1: Hard and soft tissue components 


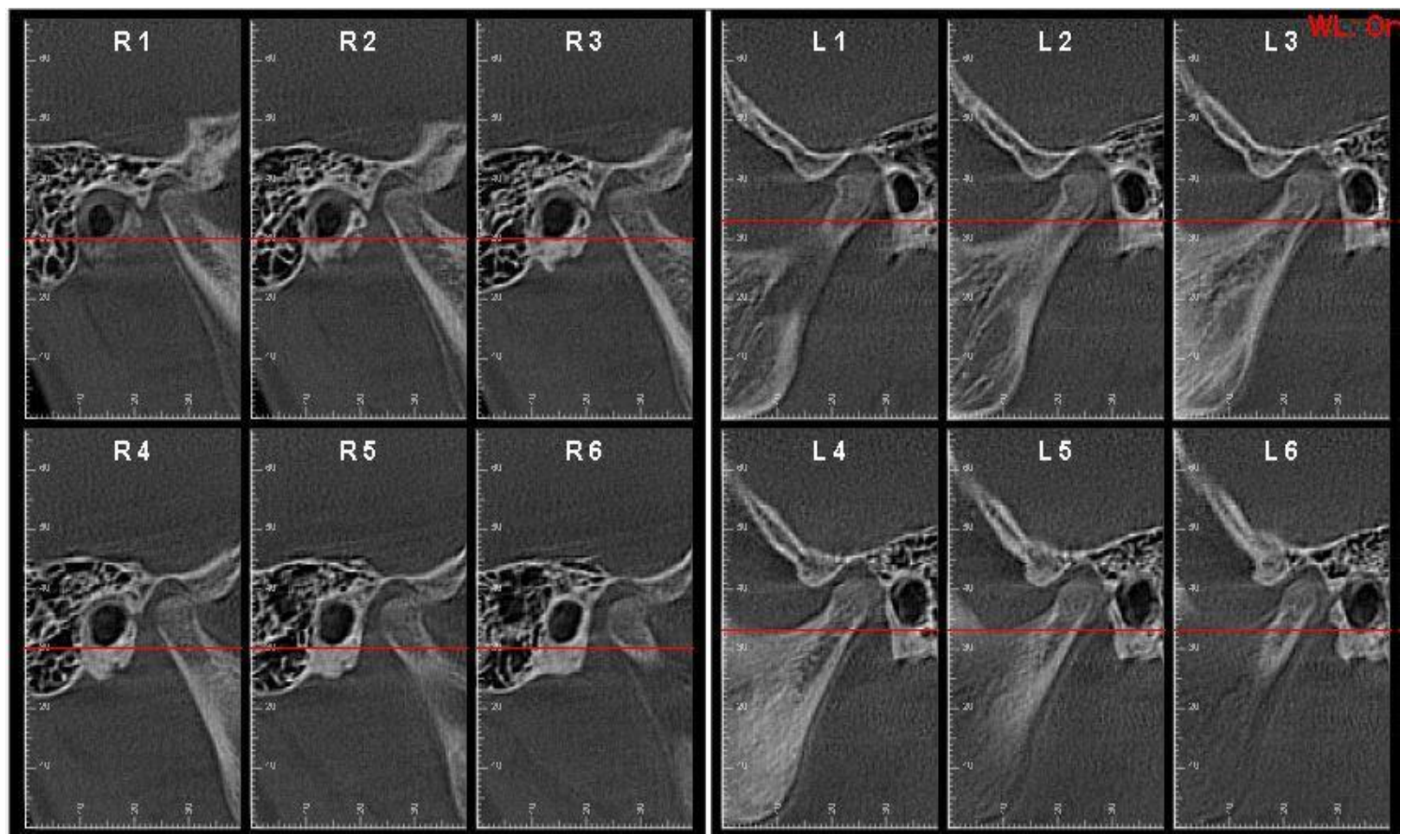

Fig. 2: The six cuts shown in detail of right and left condyle

\section{Condylar position}

The vertical reference plane is kept at the anterior border of the external auditory meatus, then a tangent is drawn to the posterior curve of the condyle head. The linear distance between the vertical plane and the tangent is then measured. Measurements were made on both right and left condyles (Fig. 3).

\section{Condylar height}

The horizontal reference plane is kept at the inferior border of the external auditory meatus. The condylar height is measured as the distance from the maximum height of the curve on the superior portion of the condyle to the horizontal reference plane. Measurements were made on both right and left condyles (Fig. 3).

\section{Condylar width}

The vertical reference plane is kept at the posterior portion of the condyle head. A tangent is drawn to the anterior curve of the condyle head. The width is then measured. Measurements are made on both right and left condyles (Fig. 3).

\section{Posterior, superior and anterior joint spaces}

Tangents are drawn at the posterior and anterior curvature of condylar head. Perpendicular lines are drawn from these tangents extending to the glenoid fossa. These perpendicular distances assess the posterior and anterior joint spaces of the TMJ. The perpendicular distance from the tangent on the most superior point on the condylar head to the glenoid fossa measures the superior joint space. Measurements are made on both right and left condyles (Fig.3).

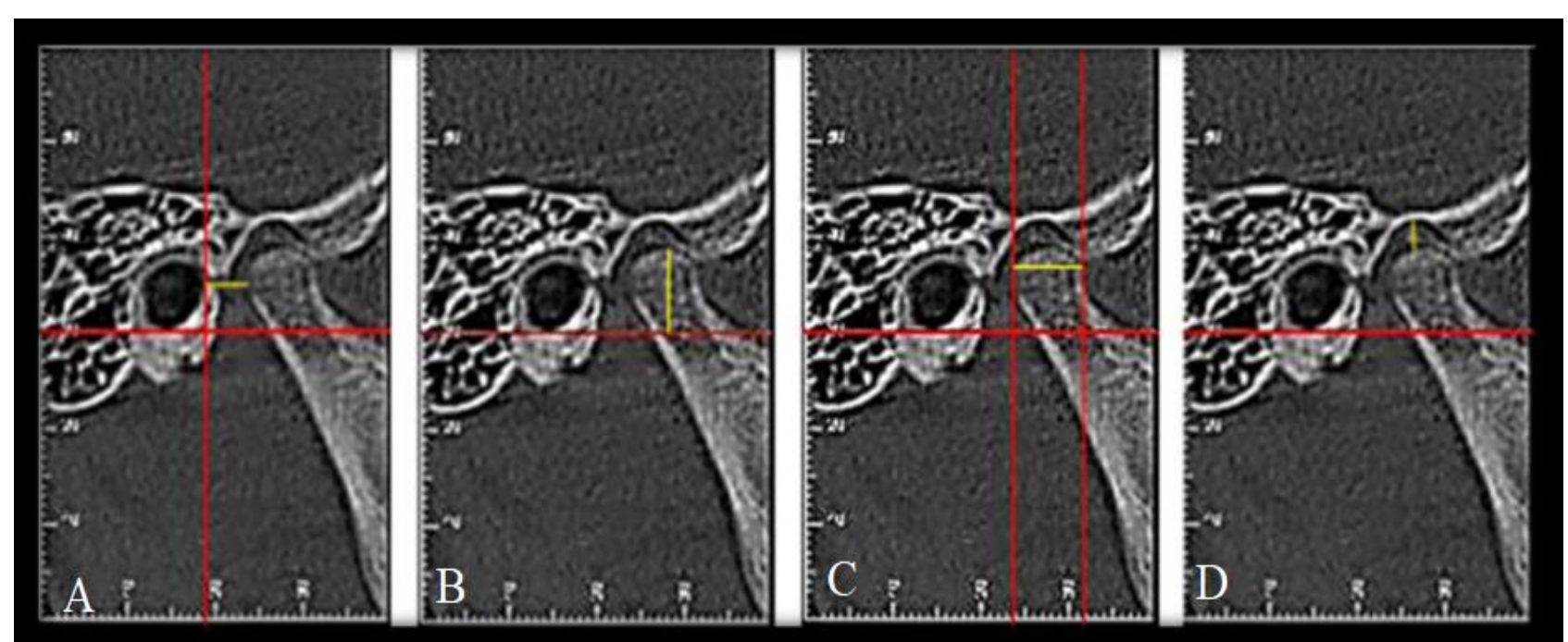

Fig. 3: Measurement-(A) Condylar position (B) Condylar height (C) Condylar width (D) Posterior, superior and anterior articular space 


\section{RESULTS}

Table 1: Comparison of the changes in TMJ structures after mandibular advancement

\begin{tabular}{|c|c|c|c|c|c|c|c|c|c|c|c|c|}
\hline \multirow[t]{2}{*}{ Appliance } & \multicolumn{2}{|c|}{$\begin{array}{l}\text { Condylar } \\
\text { Height }\end{array}$} & \multicolumn{2}{|c|}{$\begin{array}{l}\text { Condylar } \\
\text { Width }\end{array}$} & \multicolumn{2}{|c|}{$\begin{array}{l}\text { Condylar } \\
\text { Position }\end{array}$} & \multicolumn{2}{|c|}{$\begin{array}{l}\text { Posterior } \\
\text { Joint Space }\end{array}$} & \multicolumn{2}{|c|}{$\begin{array}{l}\text { Superior } \\
\text { Joint Space }\end{array}$} & \multicolumn{2}{|c|}{$\begin{array}{l}\text { Anterior } \\
\text { Joint Space }\end{array}$} \\
\hline & Right & Left & Right & Left & Right & Left & Right & Left & Right & Left & Right & Left \\
\hline Twin Block & $\sqrt{ }$ & $\sqrt{ }$ & $\Uparrow$ & 凹 & 介 & 凹 & $\sqrt{ }$ & $\sqrt{ }$ & $\sqrt{ }$ & $\sqrt{l}$ & $\sqrt{l}$ & $\sqrt{ }$ \\
\hline PowerScope & 凹 & 凹 & 凹 & 凹 & 凹 & 凹 & 凹 & 凹 & $\sqrt{l}$ & $\sqrt{l}$ & $\sqrt{1}$ & $\sqrt{ }$ \\
\hline $\begin{array}{l}\text { Forsus } \\
\text { Fatigue } \\
\text { Device }\end{array}$ & 凹 & 凹 & 凹 & 凹 & 介 & 介 & 凹 & 凹 & $\sqrt{ }$ & $\sqrt{ }$ & $\sqrt{ }$ & $\sqrt{ }$ \\
\hline
\end{tabular}

\section{Twin Block}

With the Twin Block appliance there is a decrease in condyle height, posterior joint spaces, superior joint

spaces and anterior joint spaces. The condylar width increased (refer to Fig. 4).

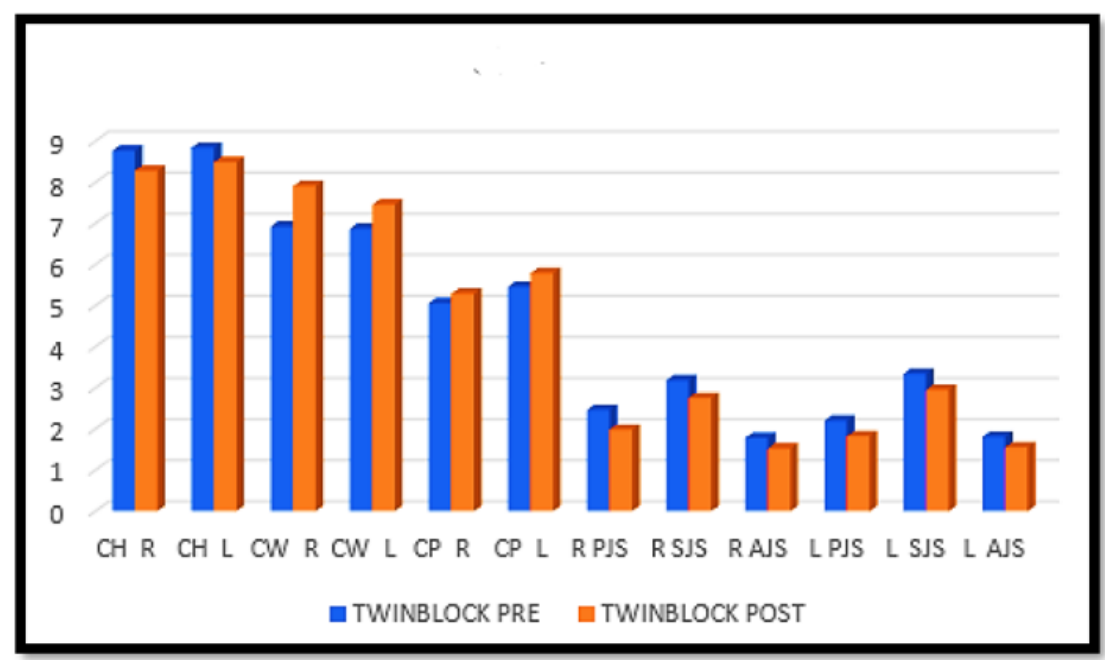

Fig. 4: Chart representing the changes in the various parameters used; pre and post Twin Block treatment

\section{Power Scope}

After intervention with Power Scope, an increase in condyle height, condylar width and the posterior joint spaces was seen with a decrease in superior and anterior joint spaces (Refer to Fig. 5).

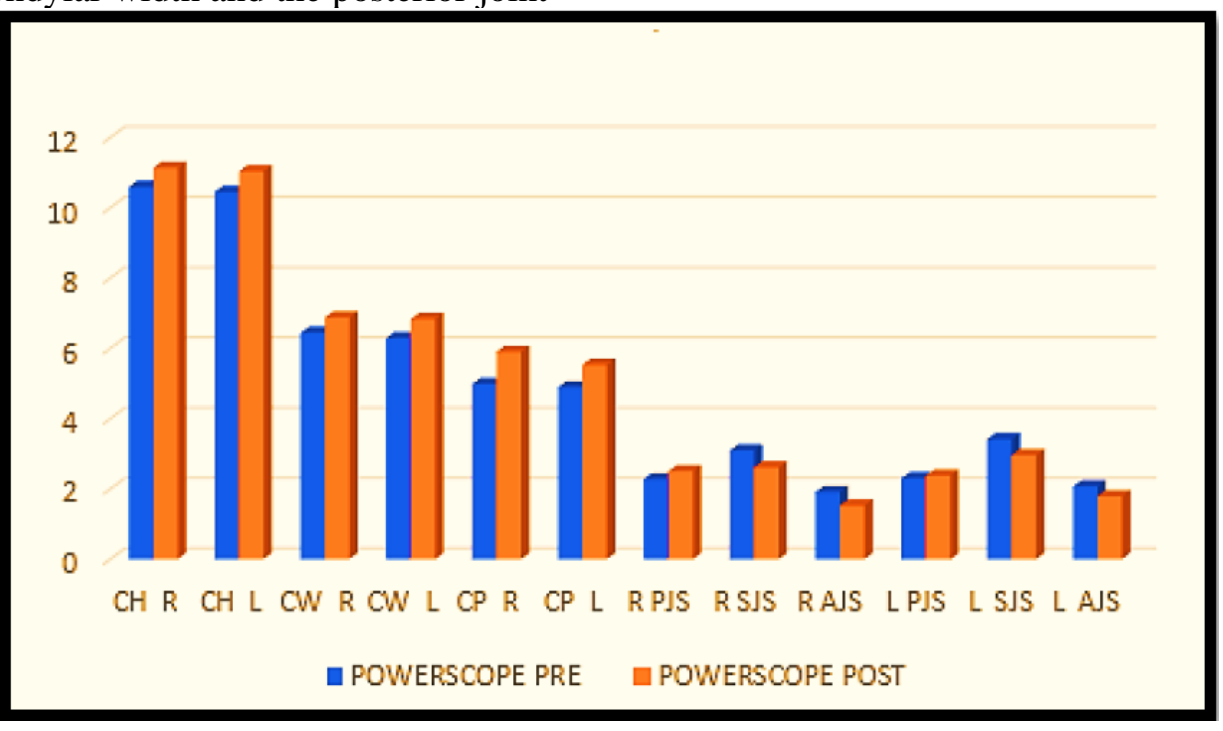

Fig.5: Chart representing the changes in the various parameters used; pre and post Power Scope treatment 


\section{Forsus Fatigue Resistant Device}

With the Forsus Fatigue Resistant Device an increase in condylar height, condylar width and posterior joint spaces was seen with a decrease in the superior and anterior joint spaces (Fig. 6).

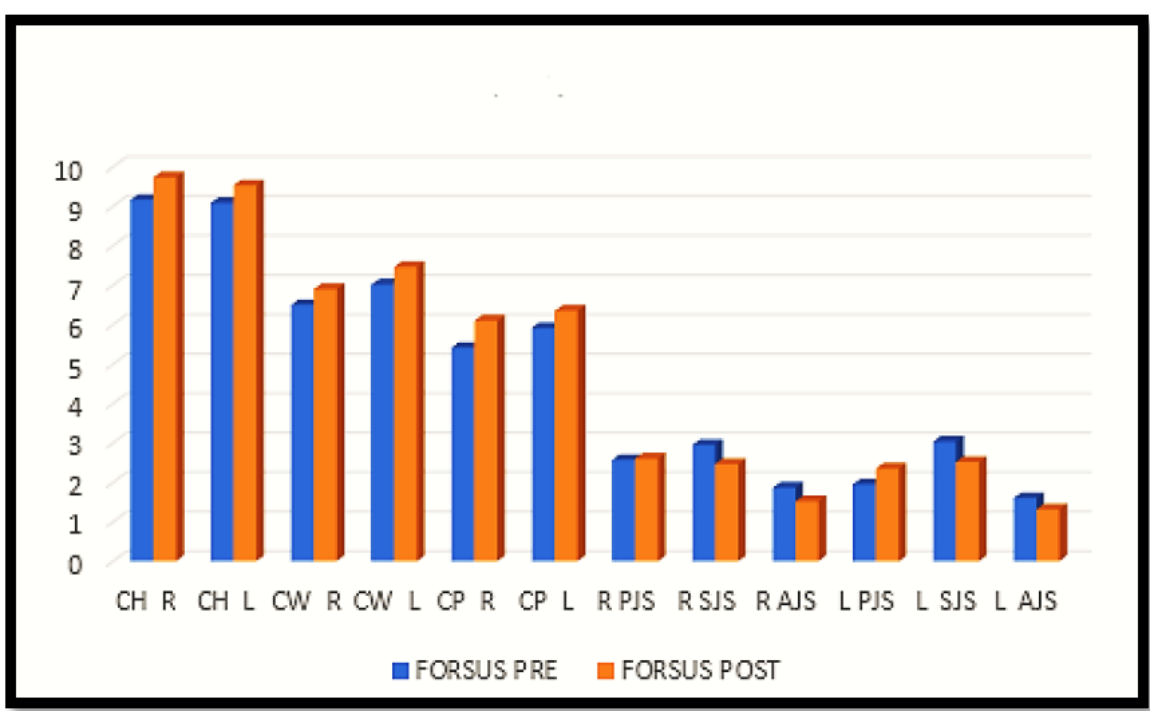

Fig. 6: Chart representing the changes in the various parameters used; pre and post Forsus Fatigue Resistant Device treatment

\section{DISCUSSION}

In the treatment of patients with class II malocclusion with mandibular retrognathia, different myofunctional or fixed functional appliances have been used to enhance or redirect the growth of the mandible to correct the skeletal discrepancy. These various functional appliances displace the condyle in the glenoid fossa and stimulate the condylar cartilage growth.

The present study evaluates the changes in TMJ structures comparing three different functional appliances using CBCT, which has not been assessed yet in literature, best known to our knowledge. With the Twin Block appliance, there was a decrease in condylar height on the right and left side, but only the right side was statistically significant. An increase in condylar width on both the right and left side was evident but was only statistically significant on the right side. There was evidence of anterior repositioning of the condyle post-treatment on both the right and left sides but were not significant statistically on either side. There was a decrease in posterior, anterior and superior joint spaces on both right and left sides which was found to be statistically significant on both sides. These changes can be due to the remodelling of glenoid fossa along with bone deposition that occurs on the posterio-superior part of the condylar head.

With the Power Scope and Forsus Fatigue Resistant Devices, there was an increase in condylar height, which was statistically significant on both sides, an increase in condylar width was seen on both sides only statistically significant on the right side. An increase in posterior joint spaces was seen but was statistically significant on the left side only and a decrease in anterior joint space that was statistically significant on both sides. There was an increase in condylar height and decrease in superior joint space which can be explained by bone deposition or progressive remodelling that involves the proliferation of tissue. There was also an increase in posterior joint space and decrease in anterior joint space due to the anterior repositioning of the condyle.

In all three appliances, anterior repositioning of the condyle has been observed. All adaptive remodelling changes are time-dependent, but if the changes are too rapid, degenerative remodelling may take place instead. Age is a factor related to this process. Adaptive processes are seemingly quite adequate during the developmental period and remain so well into adult life, but in later years the decrease in response to functional appliance therapy suggests a serious decline in adaptive and regenerative capacity.

Previously, many studies have analysed TMJ changes using conventional cephalograms, MRI or computed tomography. A study conducted on the TMJ by Pancherz, using radiographs taken before and after Herbst treatment revealed unchanged condylar relationships (8). Paulson insisted that the clinical consequences of the induced changes were because of accelerated growth of the condyles and a change in the direction of the condyles during Herbst treatment, in most cases, a more sagittal direction (9). Condylar remodelling was selective in amount and the effect of Herbst treatment in adulthood showed that the morphology of the condyle may no longer return to its original size and shape after unloading. The described biomechanically induced modelling and remodelling of TMJ condyles cannot occur without a potential growth of the cartilage, i.e., hypertrophic chondrocytes are still present in the fibrocartilage. Arruda et al., concluded that during a 12-month 
treatment period with the Herbst appliance, only a mild change in the position of the articular disc occurred and these changes were within normal physiological limits (10). Serbesis and Pancherz (11) conducted a study to compare the effectiveness of class II elastics with bonded Tip-Edge brackets and fixed functional appliance in bringing changes in TMJ and chin position. The study found fixed functional appliances to be more effective in bringing sagittal changes in TMJ growth and chin position. The study also detailed that the mandibular advancement procedure with the functional appliance resulted in condylar growth stimulation in a posterior direction and glenoid fossa remodelling, thus moving the condyle anteriorly in relation to the skull and also shifting chin forwards (11). Arici et al., conducted a computed tomography study to test the hypothesis that fixed functional appliances brings changes in the condylar position in glenoid fossa and results of this study concluded that volume of joint spaces changed and also condyle moved to more posterior position in the glenoid fossa in the study group (12). Chavan et $a l$., conducted a magnetic resonance imaging (MRI) study on changes in the TMJ following Twin Block and Bionator appliance therapy, and MRI demonstrated that a translation of the mandibular condyle occupied a more anterior position in the fossa, to its pre-treatment position and also, disclosed the movement of the disc posteriorly in relation to the condyle (13). Wadhawan et al., documented a study to evaluate the changes in the condyle-glenoid fossa (CGF) complex and the positional variations of the glenoid fossa after the use of removable functional appliance and at the end of completion of fixed appliance therapy; Forward condylar position as well as articular disc retrusion with respect to the condylar head was reported by them and they also emphasised that, even though there is forward relocation of the condyle-glenoid fossa, their internal rearrangement returns to a pre-treatment position(14).

Aras et al., reported drastic change in an articular disc position and showed a tendency to position disc progressively in relation to the condyle. The MRI revealed that the condyle-fossa relationship remains unaffected by the Forsus fatigue resistant device treatment. This might be because of appositional growth at condyle and glenoid fossa. However, there was no clear evidence of remodelling at the condyle and the glenoid fossa (15).

A study to evaluate the condylar response to functional appliance treatment with Twin Block using CBCT was conducted by Yildirima et al., The study was done on 30 patients using $60 \mathrm{CBCT}$ images (30 pre-treatment and 30 post-treatment) that had been obtained previously to evaluate $3 \mathrm{D}$ volumetric changes in the posterior airway space of patients with a retrognathic mandible, treated with Twin Block functional appliance. These images belonged to 16 male subjects with a mean age of 12 years and 14 female subjects with a mean age of 12.5 years. They concluded that CBCT can be used to successfully evaluate the condylar response to functional orthopaedic treatment and use of twin-block appliance increases condylar volume, length of the mandible and intercondylar distance thereby motivating growth of the condyle in an upward and backward direction (16). No studies have been conducted so far evaluating and comparing structural changes of the TMJ following treatment with fixed functional appliances such as the Power Scope and Forsus Fatigue Resistant Device in accordance with the vast literature already present that had been researched.

In assessing the efficacy of three different functional appliances in bringing changes to the TMJ structures, Twin Block is considered to be the most efficient (Table 2). According to Baccetti et al optimal timing for the correction of skeletal class II malocclusion by functional appliance is during or shortly after the onset of the pubertal peak in growth velocity (17). As the mean age of patients were within the optimal timing, the Twin Block was more efficient in bringing about TMJ changes as compared to the power scope and Forsus Fatigue Resistant Device. Correlating the findings of our study with the studies cited above, clearly shows that functional appliance therapy results in several favourable changes in the TMJ and its surroundings.

Table 2: Comparison of the changes in TMJ structures bought by Twin Block, Power Scope, Forsus Fatigue Device

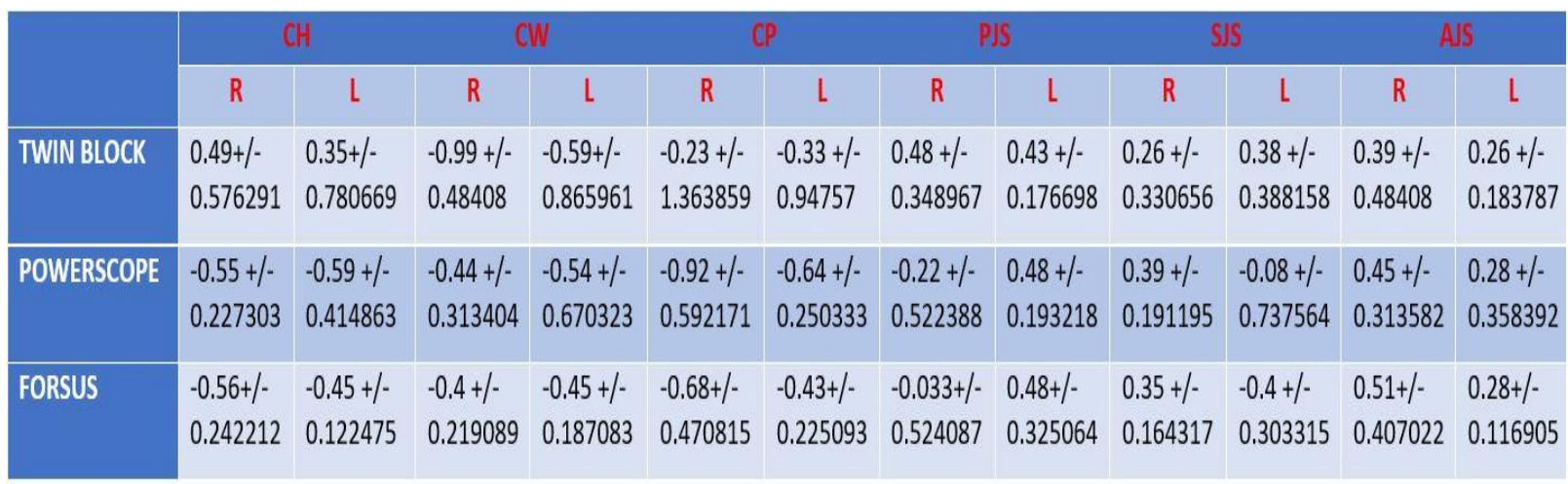




\section{CONCLUSION}

The study showed that in subjects treated with Twin Block a decrease in condylar height and articular joint spaces was seen but an increase in condylar width with anterior positioning of the condyle also was observed. In subjects treated with Power Scope and Forsus Fatigue Resistant Device there was an increase in condylar height, condylar width and posterior joint space, an anterior positioning of the condyle with a decrease in the anterior and superior joint spaces. In this study the Twin Block appliance was found to be more efficient in bringing about changes in TMJ structures.

\section{CONFLICT OF INTEREST}

Authors declare no conflict of interest.

\section{REFERENCES}

1. Bishara, S. E., Ziaja, R. R. Functional appliances: A review. Am. J. Orthod. Dentofacial Orthop. 1989; 95(3): 250-258.

2. Popowich, K., Nebbe, B., Major, P.W. Effect of Herbst treatment on temporomandibular joint morphology: a systematic literature review. Am. J. Orthod. Dentofac. Orthop. Off. Publ. Am. Assoc. Orthod. Its Const. Soc. Am. Board Orthod. 2003; 123(4): 388-394.

3. Machado, G. L. CBCT imaging - A boon to orthodontics. Saudi Dent. J. 2015; 27(1): 12-21.

4. Ghali, S., Katti, G., Shahbaz, S., Katti, C. Cone beam computed tomography: A boon for maxillofacial imaging. J. Indian Acad. Oral Med. Radiol. 2017; 29(1): 30.

5. Ngan, D. C. S., Kharbanda, O. P., Geenty, J. P., Darendeliler, M. A. Comparison of radiation levels from computed tomography and conventional dental radiographs. Aust. Orthod. J. 2003; 19(2): 67-75.

6. Halazonetis, D. J. From 2-dimensional cephalograms to 3dimensional computed tomography scans. Am. J. Orthod. Dentofac. Orthop. Off. Publ. Am. Assoc. Orthod. Its Const. Soc. Am. Board Orthod. 2005; 127(5): 627-637.

7. Swennen, G. R. J., Schutyser, F. Three-dimensional cephalometry: spiral multi-slice vs cone-beam computed tomography. Am. J. Orthod. Dentofac. Orthop. Off. Publ. Am. Assoc. Orthod. Its Const. Soc. Am. Board Orthod. 2006; 130(3): 410-416.

8. Pancherz, H. The Herbst appliance--its biologic effects and clinical use. Am. J. Orthod. 1985; 87(1): 1-20.

9. Paulsen, H. U. Morphological changes of the TMJ condyles of 100 patients treated with the Herbst appliance in the period of puberty to adulthood: a long-term radiographic study. Eur. J. Orthod. 1997; 19(6): 657-668.

10. Aidar, L. A., Abrahão, M., Yamashita, H. K., Dominguez, G. C. Herbst appliance therapy and temporomandibular joint disc position: a prospective longitudinal magnetic resonance imaging study. Am. J. Orthod. Dentofac. Orthop. Off. Publ. Am. Assoc. Orthod. Its Const. Soc. Am. Board Orthod. 2006; 129(4): 486-496.

11. Serbesis-Tsarudis, C., Pancherz, H. "Effective" TMJ and chin position changes in Class II treatment. Angle Orthod. 2008; 78(5): 813-818.

12. Arici, S., Akan, H., Yakubov, K., Arici, N. Effects of fixed functional appliance treatment on the temporomandibular joint. Am. J. Orthod. Dentofac. Orthop. Off. Publ. Am. Assoc. Orthod. Its Const. Soc. Am. Board Orthod. 2008; 133(6): 809-814.

13. Chavan, S. J., Bhad, W. A., Doshi, U. H. Comparison of temporomandibular joint changes in Twin Block and Bionator appliance therapy: a magnetic resonance imaging study. Prog. Orthod. 2014; 15(1): 57.
14. Wadhawan, N., Kumar, S., Kharbanda, O. P., Duggal, R., Sharma, R. Temporomandibular joint adaptations following two-phase therapy: an MRI study. Orthod. Craniofac. Res. 2008; 11(4): 235-250.

15. Aras, A., Ada, E., Saracoğlu, H., Gezer, N. S., Aras, I. Comparison of treatments with the Forsus fatigue resistant device in relation to skeletal maturity: a cephalometric and magnetic resonance imaging study. Am. J. Orthod. Dentofac. Orthop. Off. Publ. Am. Assoc. Orthod. Its Const. Soc. Am. Board Orthod. 2011; 140(5): 616-625.

16. Yildirim, E., Karacay, S., Erkan, M. Condylar response to functional therapy with Twin-Block as shown by cone-beam computed tomography. Angle Orthod. 2014; 84(6): 10181025.

17. Baccetti, T., Franchi, L., Toth, L. R., McNamara, J. A. Treatment timing for Twin-block therapy. Am. J. Orthod. Dentofac. Orthop. Off. Publ. Am. Assoc. Orthod. Its Const. Soc. Am. Board Orthod. 2000; 118(2): 159-170. 